\title{
COMPORTAMIENTO CLÍNICO DE LA INFECCIÓN Y ENFERMEDAD CAUSADA POR MICOBACTERIAS NO TUBERCULOSAS EN LATINOAMÉRICA: REVISIÓN DE ALCANCE
}

\author{
Arturo D. Mora@1,a, Santiago Giraldo@1,a, Diana A. Castillo@1,b, Beatriz E. Ferro@1,c \\ 1 Universidad Icesi, Facultad de Ciencias de la Salud, Cali, Colombia. \\ a Médico; ${ }^{\text {b }}$ odontóloga, especialista en docencia universitaria, magíster en Epidemiología; ${ }^{\mathrm{c}}$ bacterióloga y laboratorista \\ clínico, doctora en Microbiología Médica.
}

\section{RESUMEN}

Cada vez son más frecuentes los reportes de aislamientos y enfermedades producidas por micobacterias no tuberculosas (MNT). Esta revisión de alcance describe el comportamiento epidemiológico y clínico de la infección y enfermedad por MNT en Latinoamérica. Se realizó la búsqueda en las bases de datos MEDLINE vía OVID, Embase y LILACS. Después de la depuración, se incluyeron 44 artículos que representaron una población global de 2826 sujetos, a quienes se les diagnosticó infección y enfermedad por MNT; la mayoría de las investigaciones incluyeron sujetos de Brasil y Colombia (75\%); los estudios transversales fueron los más frecuentes (36,6\%), el sexo masculino fue el más afectado (61,3\%), mientras que la mediana de edad fue 40,1 años. En 37 artículos se reportó enfermedad por MNT, siendo la localización extrapulmonar (54\%) la más frecuente; las principales comorbilidades fueron las enfermedades pulmonares, VIH/sida, fibrosis quística, diabetes y desnutrición, reportadas en 13 estudios; en 15 artículos se reportó tuberculosis previa al evento por MNT. En 12 artículos se evidenciaron procedimientos estéticos; en tres, procedimientos clínicos previos. Se reportó variedad de especies de MNT, siendo Mycobacterium avium (52\%), M. abscessus (34\%), M. chelonae (18\%), M. fortuitum (16\%) y M. kansasii $(9,1 \%)$ las más frecuentes. El método más usado para diagnosticar e identificar la enfermedad por MNT fue el cultivo, recientemente se agregaron también las pruebas moleculares. La literatura científica latinoamericana sobre la infección/enfermedad por MNT es escasa. Es apremiante conducir estudios de frecuencia e impacto clínico y fortalecer la capacidad diagnóstica y las redes de organizaciones existentes enfocadas al estudio de micobacterias para conocer la verdadera morbimortalidad asociada a las MNT en Latinoamérica.

Palabras clave: Micobacterias no tuberculosas; Latinoamérica; Signos y Síntomas; Diagnóstico; Tratamiento (fuente: DeCS BIREME)

\section{CLINICAL BEHAVIOR OF INFECTION AND DISEASE CAUSED BY NON-TUBERCULOUS MYCOBACTERIA IN LATIN AMERICA: SCOPING REVIEW}

\begin{abstract}
Citar como: Mora AD, Giraldo S, Castillo DA, Ferro BE. Comportamiento clínico de la infección y enfermedad causada por micobacterias no tuberculosas en Latinoamérica: Revisión de alcance. Rev Peru Med Exp Salud Publica. 2021;38(2):31825. doi: https://doi.org/10.17843/ rpmesp.2021.382.6108
\end{abstract}

\section{Correspondencia:}

Beatriz E. Ferro; Calle 18 \# 122-135, Cali, Colombia; beferro@icesi.edu.co

Recibido: 30/06/2020

Aprobado: 12/05/2021

En Línea: 22/06/2021

\begin{abstract}
Reports of infection and/or disease caused by non-tuberculous mycobacteria (NTM) are becoming increasingly frequent. This scope review describes the epidemiological and clinical trend of infection/disease caused by NTM in Latin America. OVID MEDLINE, Embase and LILACS databases were explored for relevant articles. After filtering, we included 44 articles, representing an overall population of 2,826 subjects diagnosed with NTM infection and disease; the majority of the publications included subjects from Brazil and Colombia (75\%), cross-sectional studies were the most common (36.6\%), most subjects were male $(61.3 \%)$ and the median age of subjects was 40.1 years. Disease by NTM was reported in 37 publications, extrapulmonary presentation was the most frequent (54\%), main comorbidities were other pulmonary diseases, HIV, cystic fibrosis, diabetes and malnutrition, as reported in 13 studies; tuberculosis diagnosis previous to NTM disease was reported in 15 articles. Aesthetic procedures were reported in 12 articles while clinical procedures were reported in 3 articles. Several NTM species were reported, being Mycobacterium avium (52\%), M. abscessus (34\%), M. chelonae (18\%), M. fortuitum (16\%) and M. kansasii (9.1\%) the most frequent. Culture and molecular testing were the main methods for diagnosis and identification. Scientific literature on NTM from Latin American countries is scarce. There is an urgent need to conduct studies on the frequency and clinical impact of NTM infections, in order to accurately identify the current morbidity and mortality associated with NTM in Latin American. It is also important to strengthen the local diagnostic capacity and the existing networks focused on studying NTM.
\end{abstract}

Keywords: Nontuberculous Mycobacteria; Latin America; Signs and symptoms; Diagnosis; Treatment (source: MeSH NLM) 


\section{INTRODUCCIÓN}

En los últimos años, los reportes de enfermedades producidas por micobacterias no tuberculosas (MNT) han aumentado ${ }^{(1)}$. Estos microorganismos, pertenecientes al género Mycobacterium, son comunes en el aire, agua y suelo. Por su importancia clínica destacan las especies Mycobacterium avium (complejo MAC), M. kansasii, M. xenopi, M. malmoense y M. abscessus ${ }^{(2)}$. Aunque la información sobre su transmisión es aún controversial, cada vez se conoce más sobre los potenciales mecanismos de infección y patogenia. Las enfermedades producidas por MNT pueden dividirse de acuerdo a su localización pulmonar y extrapulmonar; la enfermedad pulmonar radiológicamente se presenta más comúnmente como enfermedad fibrocavitaria o bronquiectasia nodular. La localización extrapulmonar varía entre linfadenitis, infección de piel, tejidos blandos y hueso, y la diseminada es común en personas que viven con VIH ${ }^{(2)}$. A pesar de que las MNT pueden infectar y causar enfermedades tanto a individuos inmunocompetentes como a inmunocomprometidos, estos últimos tienen más riesgo de morbimortalidad; suelen encontrarse casos de concomitancia con otro tipo de morbilidades, como coinfección con VIH, cáncer, enfermedad pulmonar obstructiva crónica (EPOC), fibrosis quística, entre otras ${ }^{(3)}$.

En los países desarrollados hay un aumento en la frecuencia de las MNT, por lo cual existe un creciente interés en investigar y publicar de artículos científicos sobre el tema ${ }^{(1-4)}$. En contraste, en Latinoamérica los reportes sobre MNT son escasos, lo cual puede deberse a una baja frecuencia o a la posible subestimación de enfermedades producidas por estos microorganismos. La tuberculosis (TB) es más frecuente y más conocida en la región, y ante la dificultad de contar con herramientas de diagnóstico o confirmación, la sospecha de TB prevalece sobre la de enfermedad pulmonar por MNT, lo que explicaría en parte un subdiagnóstico ${ }^{(5,6)}$. De otro lado, las revisiones disponibles sobre MNT provienen en su mayoría de países desarrollados, algunas de las cuales, incluyen información sobre países de la región ${ }^{(1,7-9)}$, pero no hay revisiones propias de Latinoamérica.

Con el objetivo de explorar el perfil epidemiológico y clínico de la infección y enfermedad por MNT en países de Latinoamérica, realizamos una revisión de alcance de la literatura científica disponible.

\section{BÚSQUEDA Y SELECCIÓN DE EVIDENCIA}

Se revisó el alcance de la literatura relacionada con la infección o enfermedad por MNT en Latinoamérica utilizando la guía PRISMA-ScR ${ }^{(10)}$. El proceso se realizó en cuatro fases: la fase inicial, donde se identificaron artículos sobre el comportamiento clínico de las MNT en Latinoamérica (eliminando los duplicados); la fase de filtración, donde se excluyeron artículos por título y resumen, como los reportes sobre pacientes con úlcera de Buruli e investigaciones preclínicas; la fase de elegibilidad, donde se revisó el texto completo y se excluyeron los artículos cuya metodología o población no correspondía al alcance de la revisión; y la fase de inclusión, donde se seleccionaron los artículos para el análisis final. Se realizó la búsqueda en las bases de datos MEDLINE vía OVID, Embase y LILACS, sin restricción de año hasta enero de 2019, en inglés y español. La estrategia de búsqueda se realizó con lenguaje controlado y no controlado, según la base de datos, con palabras clave conforme a la estrategia PICO. Una de las estrategias de búsqueda se presenta en el material suplementario.

Los resultados de las búsquedas se verificaron de forma cruzada con el fin de eliminar los duplicados. Dos investigadores extrajeron los datos de manera independiente, utilizando un formato en Microsoft Excel, de acuerdo a las variables propuestas: año de publicación, país, tipo de estudio, TB concomitante o previa, comorbilidades, cirugía o procedimiento estético, coinfección por VIH, sexo, edad, número de casos, localización (pulmonar o extrapulmonar), hallazgos radiológicos, diagnóstico, especie de micobacteria, tratamiento y desenlace. Los desacuerdos entre los dos autores se resolvieron mediante una socialización con un tercero hasta llegar a un consenso y se realizó un análisis cualitativo de la información. Con respecto a los diseños de estudio, estos se agruparon como reporte de caso, serie de casos, transversales, cohorte, casos y controles.

\section{RESULTADOS}

Con la estrategia de búsqueda realizada se obtuvieron 168 artículos; se eliminaron 12 por estar duplicados. Posteriormente se eliminaron 88 artículos, aplicando los criterios de selección por título y resumen; se eliminaron 24 luego de leer el texto completo. Los artículos fueron revisados uno a uno en búsqueda de las variables de interés, el proceso de selección puede observarse en la Figura 1. En total se revisaron 44 artículos publicados desde 1993 hasta enero de $2019^{(7,11-53)}$. El tamaño de muestra reportado en los artículos, osciló entre reportes de caso y 730 sujetos a quienes se les aisló al menos una MNT, para un total de 2826 sujetos incluidos (Tabla 1).

En cuanto al tipo de estudio identificado para los artículos, 36,4\% (16/44) fue transversal; 20,5\% (9/44), reportes de caso y se encontró esta misma frecuencia para estudios de cohorte; 13,6\%, (6/44) casos y controles; y 9,1\% (4/44), serie de casos. El país de origen más frecuente de dichos estudios fue Brasil, lo cual corresponde a un 54,5\% (24/44), seguido de Colombia con un 20,5\% (9/44); Argentina y Venezuela con 6,8\% (3/44); Ecuador 4,5\% (2/44); México, Perú y Uruguay con $2,3 \%(1 / 44)$ cada uno (Tabla 1 y material suplementario). 
Tabla 1. Principales características encontradas en los estudios incluidos en la revisión

\begin{tabular}{|c|c|c|c|}
\hline Característica & Descripción & $\mathbf{n}$ & Unidad de análisis \\
\hline Infección o enfermedad por MNT & Sujetos de estudio & 2826 & Sujetos de estudio \\
\hline \multirow[t]{8}{*}{ País de origen } & Brasil & 24 & Artículos \\
\hline & Colombia & 9 & \\
\hline & Argentina & 3 & \\
\hline & Venezuela & 3 & \\
\hline & Ecuador & 2 & \\
\hline & México & 1 & \\
\hline & Perú & 1 & \\
\hline & Uruguay & 1 & \\
\hline \multirow[t]{2}{*}{ Sexo } & Masculino & 641 & Sujetos de estudio \\
\hline & Femenino & 404 & \\
\hline Edad (años) & Mediana & 40 & Sujetos de estudio \\
\hline \multirow[t]{2}{*}{ Factores de riesgo } & Reportados & 13 & Artículos \\
\hline & No reportados & 31 & \\
\hline \multirow[t]{3}{*}{ Enfermedad por MNT } & Pulmonar & 14 & Artículos \\
\hline & Extrapulmonar & 20 & \\
\hline & Pulmonar y extrapulmonar & 3 & \\
\hline \multirow{2}{*}{$\begin{array}{l}\text { Mecanismo para identificación de MNT } \\
\text { reportado por el laboratorio }\end{array}$} & Cultivo y pruebas bioquímicas & 9 & Artículos \\
\hline & Cultivo y pruebas moleculares & 35 & \\
\hline
\end{tabular}

MNT: micobacterias no tuberculosas.

No se encontró información sobre las variables sociodemográficas sexo y edad de todos los sujetos de estudio en los artículos. De los 1045 sujetos con infección o enfermedad por MNT, con información sobre sexo, $61,3 \%$ eran hombres; con respecto a la edad, la mediana para la población de la que se reportó información fue 40,1 años (17-70 años) (Tabla 1 y material suplementario). La incidencia solo se encontró en un estudio realizado en Brasil, el cual reportó 5-7 casos por año ${ }^{(36)}$; los estudios restantes no reportaron alguna medida de frecuencia de infección o enfermedad para sus poblaciones.

\section{Factores de riesgo y comorbilidades}

Con relación a los factores de riesgo y comorbilidades, se encontró que solo el 29,5\% (13/44) de los estudios documentaron su presencia. Dentro de estas, a nivel pulmonar, las bronquiectasias y el EPOC, fueron las más frecuentes, seguidas del tabaquismo ${ }^{(18,23,36)}$. Por ejemplo, en un estudio de Brasil, donde hay un total de 174 sujetos de estudio, en 38 se encontraron bronquiectasias y en $36 \mathrm{EPOC}$, además, 95 eran fumadores activos $^{\left({ }^{(3)}\right)}$. También se reportó VIH, diabetes, fibrosis quística, desnutrición y otros estados de inmunosupresión diferentes a $\mathrm{VIH} /$ sida $^{(22,36,37,46)}$. En 34,1\% (15/44) de los artículos se reportó TB previa al evento por MNT (material suplementario), en 4 pacientes se documentó infección concomitante por alguna MNT y Mycobacterium tuberculosis ${ }^{(13,15,16)}$.

\section{Cuadro clínico}

En 37 artículos se reportó enfermedad por MNT, el resto correspondió a aislamientos microbiológicos en individuos asintomáticos (infección). La enfermedad extrapulmonar fue más frecuente que la pulmonar; en 38\% (14/38) de los artículos se describen manifestaciones clínicas de origen pulmonar (Tabla 1 y material suplementario). El estudio de Matos, halló dificultad para respirar en la evaluación inicial en $58 \%$ de pacientes (11/19), fatiga en $26 \%$, hemoptisis en $21 \%$, dolor torácico en $21 \%$ y fiebre en $16 \%{ }^{(18)}$. La mayoría de los pacientes con enfermedad pulmonar de MNT tenía síntomas inespecíficos, como fiebre, pérdida de peso, sudoración, astenia y adinamia. No obstante, solo en 8 artículos se reportaron hallazgos imagenológicos a nivel pulmonar, siendo las atelectasias y cavitaciones las más frecuentes $(18,28,35,36,41,44,51,53)$. En contraste, la enfermedad extrapulmonar se reportó en 54\% (20/37) de los artículos analizados, dentro de la enfermedad extrapulmonar el $85 \%$ (17/20) correspondía a localización cutánea. Tres artículos reportaron pacientes que tenían enfermedad pulmonar y pacientes con enfermedad extrapulmonar.

En 65\% (15/23) de los artículos se evidenciaron procedimientos quirúrgicos estéticos y no estéticos como factor de exposición desencadenante de enfermedad extrapulmonar. El $80 \%(12 / 15)$ de esos artículos estaba asociado a intervenciones estéticas, como mesoterapia, implantes mamarios, tatua- 


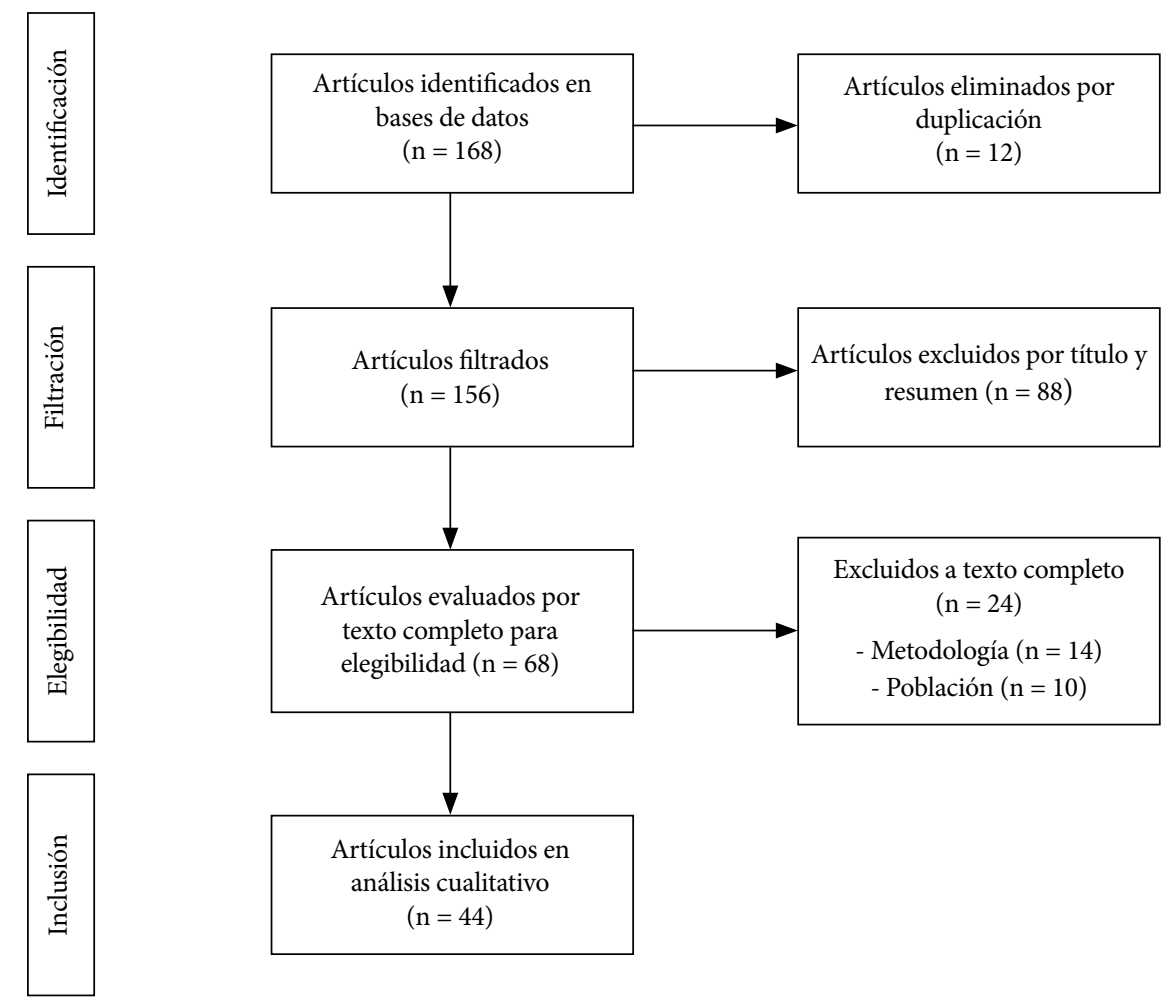

Figura 1. Diagrama de flujo del proceso de selección de artículos

jes y acupuntura. En los tres estudios restantes, se evidenció laparoscopia y artroscopia ${ }^{(27)}$, reemplazo valvular aórtico ${ }^{(32)} \mathrm{y}$ cirugías relacionadas con el sistema gastrointestinal ${ }^{(50)}$.

\section{Etiología y diagnóstico microbiológico}

Aunque se reportó una amplia diversidad de especies de MNT, las cuatro especies con mayor frecuencia de reporte fueron Mycobacterium avium en 52\% de los artículos (23/44), seguido de $M$. abscessus en $34 \%$ (15/44), M. chelonae en $18 \%$ (8/44), M. fortuitum en $16 \%$ (7/44) y M. kansasii en $9,1 \%$ (4/44) (material suplementario). Dentro de las estrategias para el diagnóstico o identificación de MNT por el laboratorio, los artículos revisados reportaron principalmente cultivo y pruebas bioquímicas, aunque en los más recientes se utilizaron también pruebas moleculares (Tabla 1 y material suplementario). En seis de los artículos se llegó al diagnóstico de enfermedad por MNT siguiendo los demás criterios de la American Thoracic Society (ATS) $(18,35,36,37,38,53)$, como ejemplo, tenemos el estudio de Matos, donde 14 de 19 pacientes cumplieron criterios diagnósticos de enfermedad pulmonar por MNT ${ }^{(18)}$. En el 45,4\% (20/44) de los artículos estudiados se reportó tratamiento; la rifampicina, el etambutol y la claritromicina fueron los antibióticos más usados, principalmente para el manejo de casos con aislamientos de MAC (material suplementario).

\section{Desenlaces}

Solo en 20 artículos se reportó seguimiento de los sujetos de estudio con un total de 722 sujetos con infección o enfermedad por MNT. Se informó que 55 sujetos $(7,6 \%)$ se curaron y $39(5,4 \%)$ murieron, aunque hubo reportes como el de Imperiale et al., en el que la tasa de mortalidad fue del 20\%, 7 personas vivían con $\mathrm{VIH} /$ sida $^{(34)}$. En el 25\% (11/44) de los artículos se reportaron recaídas, pero no informan la cantidad de sujetos que recayeron. El mismo Imperiale et al. encontró que, durante un año de seguimiento terapéutico, el $6,9 \%$ de los pacientes presentó recaída ${ }^{(34)}$.

\section{DISCUSIÓN}

Las MNT son cada vez más frecuentes en el mundo, por lo que su diagnóstico y manejo representa un reto en la práctica clínica. Este estudio describe el comportamiento clínico de las infecciones y enfermedades por MNT reportadas en Latinoamérica, a través de la revisión de 44 artículos publicados hasta enero de 2019. De los 21 países que componen Latinoamérica, solo siete publicaron artículos o fueron objeto de estudios con relación a esta temática, y se ajustaron a este enfoque metodológico. Los estudios realizados en Brasil y Colombia, representan el 75\% de la literatura revisada por los investigadores, lo cual respalda el argumento sobre la escasez de producción académica sobre MNT y la necesidad de estimar la magnitud real de este potencial problema en la región. 
La mayoría de los estudios analizados reportó enfermedad por MNT, siendo M. avium la especie que se reportó con más frecuencia. Esto se corresponde con la tendencia observada en países de Europa y Norteamérica, y en Australia. Sin embargo, en estudios con sujetos de diferentes partes del mundo se describe que $M$. avium se encuentra en la mayoría de los aislamientos, pero es en Suramérica donde en menor frecuencia se ha reportado ${ }^{(1,8,9)}$. Se evidencia la necesidad de caracterizar las especies de MNT circulantes en Latinoamérica y establecer su perfil de susceptibilidad; para esto se deben fortalecer las capacidades locales de laboratorio y recursos humanos.

Si bien la información sobre las variables sociodemográficas fue limitada, el análisis de variables como la edad y el sexo evidencia que la población de hombres adultos, en edad media, fue la más afectada. Es necesario verificar si esta misma población es la más afectada en la región, dado que contrasta con estudios en otras latitudes, por ejemplo, en el continente europeo, donde se ha evidenciado que las mujeres, generalmente mayores de 60 años, son la población más afectada por $\mathrm{MNT}^{(6)}$.

Por otro lado, la sintomatología identificada en el presente estudio, fue mayormente inespecífica, como diaforesis nocturna, pérdida de peso, astenia, adinamia y fiebre, tal como se describe en uno de los estudios ${ }^{(18)}$. Aunque dicha inespecificidad se encuentra relacionada a enfermedades como el VIH/ sida ${ }^{(54)}$, cabe anotar que en la enfermedad pulmonar es similar con la de TB, que, como se ha mencionado, es frecuente en Latinoamérica. Encontramos que en la enfermedad pulmonar las atelectasias y las cavitaciones identificadas en la radiografía y el TAC de tórax, respectivamente, fueron frecuentes ${ }^{(28,35)}$; en algunos de los estudios en los que se reportaba infección o enfermedad, los pacientes tenían antecedentes de cavitaciones, bronquiectasias y tabaquismo ${ }^{(23,47,53)}$, y en otros, algunos pacientes habían sido diagnosticados y tratados contra la $\mathrm{TB}$, previo al diagnóstico de $\mathrm{MNT}{ }^{(35,47,48)}$. Esto cobra importancia debido a la tendencia a asociar la TB con patologías crónicas y a darle tratamiento, dejando de lado posibles diagnósticos diferenciales, especialmente la enfermedad pulmonar producida por MNT ${ }^{(47)}$. Estudios prospectivos sobre la importancia clínica de los aislamientos de MNT son igualmente necesarios para identificar los determinantes de infección y enfermedad pulmonar por MNT en nuestra población latinoamericana.

La enfermedad extrapulmonar fue más frecuente que la pulmonar. Según la literatura universal, se han asociado las MNT de crecimiento rápido con la afectación de piel y tejidos blandos, como M. abscessus, $M$. fortuitum y M. chelonae ${ }^{(2,55)}$. En esta revisión, los casos de afectación de piel y tejidos blandos se relacionaron con inyecciones con lidocaína ${ }^{(12)}$ y mesoterapia con fines cosméticos, entre otros ${ }^{(21)}$. Los hallazgos clínicos consistían en signos y síntomas de inflamación local, como dolor, calor, rubor, edema, y en algunos, fiebre; y se manifes- taban desde pápulas hasta nódulos eritematosos y purulentos, y abscesos $(12,21,22,29,42)$. En Latinoamérica, las cirugías y procedimientos estéticos son ampliamente desarrollados, en comparación con el resto del mundo ${ }^{(56)}$, por lo que estos antecedentes representan uno de los factores de riesgo a tener en cuenta para la enfermedad extrapulmonar por MNT.

La enfermedad diseminada por MNT es causada principalmente por el complejo MAC ${ }^{(12)}$, y se reporta con mayor frecuencia en personas con $\mathrm{VIH}$, sobre todo en aquellas con conteo de linfocitos T CD4+ menor de 50 células $/ \mathrm{mm}^{3}{ }^{357)}$. Sus manifestaciones son atípicas: fiebre, pérdida de peso, megalias, dolor abdominal, diarrea, diaforesis nocturna y citopenias, y en la mayoría de las veces se documenta como bacteriemia ${ }^{(58,59)}$, con una supervivencia disminuida y hasta tres veces el riesgo de muerte ${ }^{(9)}$. Nuestros hallazgos sobre MNT en personas con VIH son consistentes con los reportados en la literatura científica; el principal agente etiológico reportado fue M. avium, aislado especialmente en sangre ${ }^{(11,13,16,28,46)}$. A pesar de que no en todos los estudios se reportó el conteo de linfocitos T CD4+, en uno de ellos la información se correlaciona con lo ya descrito, y se documentó el estado de micobacteriosis diseminada con un conteo menor de 50 células $/ \mathrm{mm}^{3}$ (28).

Para el diagnóstico de la enfermedad pulmonar por MNT, se usan guías oficiales ${ }^{(2,60)}$ que tienen en cuenta parámetros clínicos: los pacientes deben presentar sintomatología respiratoria y se deben haber descartado otros diagnósticos diferenciales; microbiológicos: relacionados con la obtención de al menos dos cultivos positivos en al menos dos muestras separadas de esputo o cultivo positivo de un lavado broncoalveolar o biopsia transbronquial o tras cualquier biopsia con características histológicas de MNT; y radiológicos: los nódulos, cavitaciones y bronquiectasias multifocales pulmonares son los principales hallazgos evidenciables en la radiografía de tórax o TAC pulmonar ${ }^{(2)}$. A pesar de esto, en esta revisión se evidenció un limitado seguimiento de estas guías para establecer el diagnóstico. En la gran mayoría de estudios se usaron métodos microbiológicos para el diagnóstico: Aily et al. usaron hemocultivos bifásicos ${ }^{(14)}$ o en el caso de Barreto et al., cultivos realizados en muestra obtenida de aspirado de medula ósea ${ }^{(11)}$; también se usaron métodos de detección molecular o genética como se pudo evidenciar en el caso de Pedro et al. ${ }^{(26)}$ con el uso de amplificación del gen $h s p 65$, adicional al cultivo microbiológico. Sin embargo, es prioritario aplicar las guías existentes para enfermedad pulmonar y contar con consensos clínicos y microbiológicos que permitan formular guías para orientar el diagnóstico y manejo de la enfermedad extrapulmonar por MNT.

Una de las limitaciones de esta investigación es la falta de uniformidad de los datos reportados y la escasez de información para algunas de las variables, lo cual dificulta recomendar diagnósticos y terapias a la comunidad científica en Latinoamérica. Otra limitación es no haber incluido artículos 
en portugués ni la mortalidad como una de las variables en el algoritmo de búsqueda y análisis de información. Es posible que no incluir otras bases de datos bibliográficas o literatura gris haya dejado afuera algunos estudios de interés para esta revisión.

\section{CONCLUSIONES}

La literatura científica latinoamericana sobre el comportamiento clínico de infección o enfermedad por MNT es escasa y puede subestimar la magnitud de estos eventos. Sin embargo, la revisión de 44 artículos publicados hasta enero de 2019 evidencia que, aparentemente, son más frecuentes en hombres de edad media, y que $M$. avium fue la especie más reportada. Los síntomas de enfermedad pulmonar, aunque inespecíficos, son similares con los de la TB pulmonar, enfermedad frecuente en la región, por lo que es importan-

\section{REFERENCIAS BIBLIOGRÁFICAS}

1. Hoefsloot W, van Ingen J, Andrejak C, Angeby K, Bauriaud R, Bemer P, et al. The geographic diversity of nontuberculous mycobacteria isolated from pulmonary samples: an NTM-NET collaborative study. Eur Respir J. 2013;42(6):1604-13. doi: 10.1183/09031936.00149212.

2. Griffith DE, Aksamit T, Brown-Elliott BA, Catanzaro A, Daley C, Gordin F, et al. An official ATS/IDSA statement: diagnosis, treatment, and prevention of nontuberculous mycobacterial diseases. Am J Respir Crit Care Med. 2007;175(4):367-416. doi: 10.1164/rccm.200604-571ST.

3. van Ingen J, Bendien SA, de Lange WC, Hoefsloot W, Dekhuijzen PN, Boeree MJ, et al. Clinical relevance of non-tuberculous mycobacteria isolated in the Nijmegen-Arnhem region, The Netherlands. Thorax. 2009;64(6):502-6. doi: 10.1136/thx.2008.110957.

4. Stout JE, Koh W-J, Yew WW. Update on pulmonary disease due to non-tuberculous mycobacteria. Int J Infect Dis IJID Off Publ Int Soc Infect Dis. 2016 Apr; 45:123-34. doi: 10.1016/j.ijid.2016.03.006.

5. Harding E. WHO global progress report on tuberculosis elimination. Lancet Respir Med. 2020;8(1):19. doi: 10.1016/S2213-2600(19)30418-7.

6. Prevots DR, Marras TK. Epidemiology of human pulmonary infection with nontuberculous mycobacteria: a review. Clin Chest Med. 2015;36(1):13-34. doi: 10.1016/j.ccm.2014.10.002.

7. Bax HI, van Ingen J, Dwarkasing RS, Verbon A. [Lipotourism, not without risks: a complication of cosmetic surgery abroad]. Ned Tijdschr Geneeskd. 2014;158: A7926.

8. Marras TK, Daley CL. Epidemiology of human pulmonary infection with nontuberculous mycobacteria. Clin Chest Med. 2002;23(3):553-67. doi: 10.1016/s0272-5231(02)00019-9.

9. Martín-Casabona N, Bahrmand AR, Bennedsen J, Thomsen VO, Curcio M, Fauville-Dufaux M, et al. Non-tuberculous mycobacteria: patterns of isolation. A multi-country retrospective survey. Int J Tuberc Lung Dis. 2004;8(10):1186-93.

10. Tricco AC, Lillie E, Zarin W, O’Brien KK, Colquhoun H, Levac D, et al. PRISMA Extension for Scoping Reviews (PRISMA-ScR): Checklist and Explanation. Annals of Internal Medicine. 2018;169(7):467-73. doi: 10.7326/M18-0850.

11. Barreto JA, Palaci M, Ferrazoli L, Martins MC, Suleiman J, Lorenço R, et al. Isolation of Mycobacterium avium complex from bone marrow aspirates of AIDS patients in Brazil. J Infect Dis. 1993;168(3):777-9. doi: 10.1093/infdis/168.3.777.

12. Camargo D, Saad C, Ruiz F, Ramirez ME, Lineros M, Rodriguez G, et al. Iatrogenic outbreak of M. chelonae skin abscesses. Epidemiol Infect. 1996;117(1):113-9. doi: 10.1017/s0950268800001205. te considerar la enfermedad pulmonar por MNT dentro de los posibles diagnósticos diferenciales, en presencia de una clínica compatible. Es apremiante conducir estudios de frecuencia e impacto clínico, así como fortalecer la capacidad diagnóstica y las redes existentes en micobacterias, para lograr conocer la verdadera magnitud de las MNT sobre la morbimortalidad en países de Latinoamérica.

Contribuciones de autoría: AM y SG participaron en la concepción del artículo, recolección de datos, redacción y aprobación de la versión final. DAC y BEF participaron en la concepción y diseño del artículo, análisis e interpretación de datos, revisión crítica del artículo y aprobación de la versión final.

Financiamiento: Autofinanciado.

Conflictos de interés: Los autores declaran que no existe conflicto de intereses en relación con esta publicación.

Material suplementario: Disponible en la versión electrónica de la RPMESP.

13. Crespo MP, Heli Corral R, Alzate A, Carrasquilla G, Sánchez N. [Mycobacterial infections in HIV-infected patients in Cali, Colombia]. Rev Panam Salud Publica. 1999;6(4):249-55. doi: 10.1590/s102049891999000900004.

14. Aily DC, Camargo SS, Paro HS, Passos CA, Coelho AG, Sato DN, et al. Systemic mycobacterioses in AIDS patients as determined by blood cultures on biphasic medium. Rev Argent Microbiol. 1999;31(2):53-7.

15. Murcia-Aranguren MI, Gómez-Marin JE, Alvarado FS, Bustillo JG, de Mendivelson E, Gómez B, et al. Frequency of tuberculous and non-tuberculous mycobacteria in HIV infected patients from Bogota, Colombia. BMC Infect Dis. 2001; 1:21. doi: 10.1186/1471-2334-1-21.

16. Ferreira RM, Saad MH, Silva MG, Fonseca L deS. Non-tuberculous mycobacteria I: one year clinical isolates identification in Tertiary Hospital Aids Reference Center, Rio de Janeiro, Brazil, in pre highly active antiretroviral therapy era. Mem Inst Oswaldo Cruz. 2002;97(5):725-9. doi: 10.1590/ s0074-02762002000500024.

17. Gadelha A, Accácio N, Grinzstejn B, Veloso V, da Silveira LB, Fandinho $\mathrm{F}$, et al. Low incidence of colonization and no cases of disseminated Mycobacterium avium complex infection (DMAC) in Brazilian AIDS patients in the HAART era. Braz J Infect Dis. 2002;6(5):252-7. doi: 10.1590/s1413-86702002000500006.

18. Matos ED, Santana MA, de Santana MC, Mamede P, de Lira Bezerra B, Panão ED, et al. Nontuberculosis mycobacteria at a multiresistant tuberculosis reference center in Bahia: clinical epidemiological aspects. Braz J Infect Dis. 2004;8(4):296-304. doi: 10.1590/s1413-86702004000400005.

19. Barnes AI, Rojo S, Moretto H. [Prevalence of mycobacteriosis and tuberculosis in a reference hospital, Cordoba province]. Rev Argent Microbiol. 2004;36(4):170-3.

20. Coitinho C, Brandes E, Pardiñas M, Rivas C. [Disseminated mycobacterial infections in patients with HIV/AIDS. Evaluation of blood cultures]. Rev Argent Microbiol. 2005;37(4):196-8.

21. Rivera-Olivero IA, Guevara A, Escalona A, Oliver M, Pérez-Alfonzo R, Piquero J, et al. [Soft-tissue infections due to non-tuberculous mycobacteria following mesotherapy. What is the price of beauty]. Enferm Infecc Microbiol Clin. 2006;24(5):302-6. doi: 10.1157/13089664.

22. Sañudo A, Vallejo F, Sierra M, Hoyos JG, Yepes S, Wolff JC, et al. Nontuberculous mycobacteria infection after mesotherapy: preliminary report of 15 cases. Int J Dermatol. 2007;46(6):649-53. doi: 10.1111/j.1365-4632.2007.02976. x.

23. Gutierrez M, Feola M, Lenge L, Rey R, Hoffman M. First pulmonary case reported in Argentina of infection with mycobacterium szulgai, 
a rare pathogen. J Clin Microbiol. 2007;45(9):3121-4. doi: 10.1128/ JCM.00148-07.

24. Padoveze MC, Fortaleza CM, Freire MP, Brandão de Assis D, Madalosso G, Pellini AC, et al. Outbreak of surgical infection caused by non-tuberculous mycobacteria in breast implants in Brazil. J Hosp Infect. 2007;67(2):161-7. doi: 10.1016/j.jhin.2007.07.007.

25. Zamarioli LA, Coelho AG, Pereira CM, Nascimento AC, Ueki SY, Chimara E. Descriptive study of the frequency of nontuberculous mycobacteria in the Baixada Santista region of the state of São Paulo, Brazil. J Bras Pneumol. 2008;34(8):590-4. doi: 10.1590/s1806-37132008000800008.

26. Pedro HaS, Pereira MI, Goloni MoR, Ueki SY, Chimara E. Nontuberculous mycobacteria isolated in São José do Rio Preto, Brazil between 1996 and 2005. J Bras Pneumol. 2008;34(11):950-5. doi: 10.1590/s180637132008001100010 .

27. Cardoso AM, Martins de Sousa E, Viana-Niero C, Bonfim de Bortoli F, Pereira das Neves ZC, Leão SC, et al. Emergence of nosocomial Mycobacterium massiliense infection in Goiás, Brazil. Microbes Infect. 2008;10(14-15):1552-7. doi: 10.1016/j.micinf.2008.09.008.

28. dos Santos RP, Scheid KL, Willers DM, Goldani LZ. Comparative radiological features of disseminated disease due to Mycobacterium tuberculosis vs non-tuberculosis mycobacteria among AIDS patients in Brazil. BMC Infect Dis. 2008; 8:24. doi: 10.1186/1471-2334-8-24.

29. Correa NE, Cataño JC, Mejía GI, Realpe T, Orozco B, Estrada S, et al. Outbreak of mesotherapy-associated cutaneous infections caused by Mycobacterium chelonae in Colombia. Jpn J Infect Dis. 2010;63(2):143-5.

30. Guevara-Patiño A, Sandoval de Mora M, Farreras A, Rivera-Olivero I, Fermin D, de Waard JH. Soft tissue infection due to Mycobacterium fortuitum following acupuncture: a case report and review of the literature. J Infect Dev Ctries. 2010;4(8):521-5. doi: 10.3855/jidc.718.

31. Da Mata Jardín O, Hernández-Pérez R, Corrales H, Cardoso-Leao $\mathrm{S}$, de Waard JH. [Follow-up on an outbreak in Venezuela of soft-tissue infection due to Mycobacterium abscessus associated with Mesotherapy]. Enferm Infecc Microbiol Clin. 2010;28(9):596-601. doi: 10.1016/j. eimc.2009.08.003.

32. Torres-Duque CA, Díaz C, Vargas L, Serpa EM, Mosquera W, Garzón MC, et al. Disseminated mycobacteriosis affecting a prosthetic aortic valve: first case of Mycobacterium peregrinum type III reported in Colombia. Biomedica. 2010;30(3):332-7.

33. Senna SG, Marsico AG, Vieira GB, Sobral LF, Suffys PN, Fonseca L de $S$. Identification of nontuberculous mycobacteria isolated from clinical sterile sites in patients at a university hospital in the city of Rio de Janeiro, Brazil. J Bras Pneumol. 2011;37(4):521-6. doi: 10.1590/ s1806-37132011000400015.

34. Imperiale B, Zumárraga M, Gioffré A, Di Giulio B, Cataldi A, Morcillo N. Disease caused by non-tuberculous mycobacteria: diagnostic procedures and treatment evaluation in the North of Buenos Aires Province. Rev Argent Microbiol. 2012;44(1):3-9. doi: 10.1590/S032575412012000100002.

35. Fusco da Costa AR, Falkinham JO, Lopes ML, Barretto AR, Felicio JS, Sales LH, et al. Occurrence of nontuberculous mycobacterial pulmonary infection in an endemic area of tuberculosis. PLoS Negl Trop Dis. 2013;7(7):e2340. doi: 10.1371/journal.pntd.0002340.

36. de Mello KG, Mello FC, Borga L, Rolla V, Duarte RS, Sampaio EP, et al. Clinical and therapeutic features of pulmonary nontuberculous mycobacterial disease, Brazil, 1993-2011. Emerg Infect Dis. 2013;19(3):393-9. doi: $10.3201 /$ eid/1903.120735.

37. Cortés-Torres N, González-Y-Merchand JA, González-Bonilla C, García-Elorriaga G. Molecular analysis of mycobacteria isolated in Mexican patients with different immunodeficiencies in a tertiary care hospital. Arch Med Res. 2013;44(7):562-9. doi: 10.1016/j.arcmed.2013.09.002.

38. Lima CA, Gomes HM, Oelemann MA, Ramos JP, Caldas PC, Campos $\mathrm{CE}$, et al. Nontuberculous mycobacteria in respiratory samples from patients with pulmonary tuberculosis in the state of Rondônia, Brazil. Mem Inst Oswaldo Cruz. 2013;108(4):457-62. doi: 10.1590/S00740276108042013010.
39. Zurita J, Ortega-Paredes D, Mora M, Espinel N, Parra H, Febres L, et al. Characterization of the first report of Mycobacterium timonense infecting an HIV patient in an Ecuadorian hospital. Clin Microbiol Infect. 2014;20(12):O1113-6. doi: 10.1111/1469-0691.12675.

40. Sousa PP, Cruz RC, Schettini AP, Westphal DC. Mycobacterium abscessus skin infection after tattooing--Case report. An Bras Dermatol. 2015;90(5):741-3. doi: 10.1590/abd1806-4841.20153362.

41. Campos CE, Dias CF, Unis G, de Souza Caldas PC, Redner P, de Carvalho LD, et al. First detection of Mycobacterium triplex in Latin America. Int J Mycobacteriol. 2016;5(1):89-91. doi: 10.1016/j.ijmyco.2015.09.004.

42. Torres-Coy JA, Rodríguez-Castillo BA, Pérez-Alfonzo R, DE Waard JH. Source investigation of two outbreaks of skin and soft tissue infection by Mycobacterium abscessus subsp. abscessus in Venezuela. Epidemiol Infect. 2016;144(5):1117-20. doi: 10.1017/S0950268815002381.

43. Barretto AR, Felício JS, Sales LHM, Yamada ES, Lopes ML, da Costa ARF. A fatal case of pulmonary infection by Mycobacterium colombiense in Para State, Amazon Region, Brazil. Diagn Microbiol Infect Dis. 2016;85(3):344-6. doi: 10.1016/j.diagmicrobio.2016.02.011.

44. Pedro HSP, Coelho AGV, Mansur IM, Chiou AC, Pereira MIF, Belotti NCU, et al. Epidemiological and laboratorial profile of patients with isolation of nontuberculous mycobacteria. Int J Mycobacteriol. 2017;6(3):239-45. doi: 10.4103/ijmy.ijmy_87_17.

45. Llenas-García J, Vicente J, Ruiz-García MM, Valencia-Ramírez I, Masiá M. A "lipo-tourist" with chronic cutaneous lesions after surgery in Ecuador: A diagnostic challenge. Travel Med Infect Dis. 2018;25:77-8. doi: 10.1016/j.tmaid.2018.07.013.

46. Llerena C, Valbuena A, Zabaleta AP. [Mycobacterioses identified in the National Reference Laboratory of Colombia from 2012 to 2016] Biomédica. 2018;38(0):87-94. doi: 10.7705/biomedica.v38i0.4143.

47. Carneiro MDS, Nunes LS, David SMM, Dias CF, Barth AL, Unis G. Nontuberculous mycobacterial lung disease in a high tuberculosis incidence setting in Brazil. J Bras Pneumol. 2018;44(2):106-11. doi: 10.1590/s1806-37562017000000213.

48. Beltrán-León M, Pérez-Llanos F, Sánchez L, Parra-López C, Navarrete $\mathrm{M}$, Sánchez R, et al. [Prevalence and risk factors associated to tuberculosis and non-tuberculous mycobacterial infections in HIV-positive patients in Bogotá]. Biomedica. 2018;38(1):120-7. doi: 10.7705/biomedica.v38i0.3410.

49. Marques LRM, Ferrazoli L, Chimara É. Pulmonary nontuberculous mycobacterial infections: presumptive diagnosis based on the international microbiological criteria adopted in the state of São Paulo, Brazil, 2011-2014. J Bras Pneumol. 2019;45(2):e20180278. doi: 10.1590/18063713/e20180278.

50. Baruque Villar G, de Mello Freitas FT, Pais Ramos J, Dias Campos CE, de Souza Caldas PC, Santos Bordalo F, et al. Risk Factors for Mycobacterium abscessus subsp. bolletii infection after laparoscopic surgery during an outbreak in Brazil. Infect Control Hosp Epidemiol. 2015;36(1):81-6. doi: 10.1017/ice.2014.13.

51. Aiello TB, Levy CE, Zaccariotto TR, Paschoal IA, Pereira MC, Nolasco da Silva MT, et al. Prevalence and clinical outcomes of nontuberculous mycobacteria in a Brazilian cystic fibrosis reference center. Pathog Dis. 2018;76(5). doi: 10.1093/femspd/fty051.

52. Munayco CV, Grijalva CG, Culqui DR, Bolarte JL, Suárez-Ognio LA, Quispe N, et al. Outbreak of persistent cutaneous abscesses due to Mycobacterium chelonae after mesotherapy sessions, Lima, Peru. Rev Saude Publica. 2008;42(1):146-9. doi: 10.1590/s0034-89102008000100020.

53. Monteiro JTC, Lima KVB, Barretto AR, Furlaneto IP, Gonçalves GM, Costa ARFD, et al. Clinical aspects in patients with pulmonary infection caused by mycobacteria of the Mycobacterium abscessus complex, in the Brazilian Amazon. J Bras Pneumol. 2018;44(2):93-8. doi: 10.1590/ s1806-37562016000000378.

54. Nightingale SD, Byrd LT, Southern PM, Jockusch JD, Cal SX, Wynne BA. Incidence of Mycobacterium avium-intracellulare complex bacteremia in human immunodeficiency virus-positive patients. J Infect Dis. 1992;165(6):1082-5. doi: 10.1093/infdis/165.6.1082. 
55. Lee MR, Sheng WH, Hung CC, Yu CJ, Lee LN, Hsueh PR. Mycobacterium abscessus Complex Infections in Humans. Emerg Infect Dis. 2015;21(9):1638-46. doi: 10.3201/2109.141634.

56. Heidekrueger PI, Juran S, Ehrl D, Aung T, Tanna N, Broer PN. Global aesthetic surgery statistics: a closer look. Journal of plastic surgery and hand surgery. 2017;51(4):270-4. doi: 10.1080/2000656X.2016.1248842.

57. Karakousis PC, Moore RD, Chaisson RE. Mycobacterium avium complex in patients with HIV infection in the era of highly active antiretroviral therapy. Lancet Infect Dis. 2004;4(9):557-65. doi: 10.1016/ S1473-3099(04)01130-2.
58. Grinsztejn B, Fandinho FC, Veloso VG, João EC, Lourenço MC, Nogueira SA, et al. Mycobacteremia in patients with the acquired immunodeficiency syndrome. Arch Intern Med. 1997;157(20):2359-63.

59. Phillips P, Bonner S, Gataric N, Bai T, Wilcox P, Hogg R, et al. Nontuberculous mycobacterial immune reconstitution syndrome in HIV-infected patients: spectrum of disease and long-term follow-up. Clin Infect Dis. 2005;41(10):1483-97. doi: 10.1086/497269.

60. Daley CL, Iaccarino JM, Lange C, Cambau E, Wallace RJ, Andrejak C, et al. Treatment of Nontuberculous Mycobacterial Pulmonary Disease: An Official ATS/ERS/ESCMID/IDSA Clinical Practice Guideline. Clin Infect Dis. 2020;71(4):905-13. doi: 10.1093/cid/ciaal125. 